BULLETIN OF THE

AMERICAN MATHEMATICAL SOCIETY

Volume 77, Number 6, November 1971

\title{
LEFSCHETZ FORMULA AND MORSE INEQUALITIES
}

\author{
BY EVERETT PITCHER
}

Communicated May 24, 1971

The Lefschetz fixed point formula, when applied to a gradient vector field, is the same equality as the last of the Morse inequalities of critical point theory. The latter, when applicable, contain more information than the Lefschetz fixed point formula. The Lefschetz fixed point formula has an essentially wider range of applicability, for example to maps not homotopic to the identity and hence not appearing in a deformation theory of critical points. One can ask either how to obtain more information to accompany the Lefschetz fixed point formula or how to achieve wider applicability for a theory like critical point theory.

We note that Smale has extended critical point theory to a class of vector fields which are not gradient fields and states that a similar theory applies to a class of diffeomorphisms not necessarily homotopic to the identity. (See [S].) Our approach is different in that our maps need be neither homotopic to the identity nor diffeomorphisms.

The prodecure is the following. The idea of the characteristic polynomial of an endomorphism of a finitely generated abelian group has been extended to that of a characteristic rational function. See references $[\mathrm{F}],[\mathrm{K}-\mathrm{S}]$, and $[\mathrm{M}]$ for both accounts of the ideas and earlier references to Fuller and Weil. The characteristic rational function is one of a set of invariants to be defined. The application is to downward maps, defined below, which do not necessarily have the property of being homotopic to the identity. The relations in Theorem 2 generalize the Morse inequalities and reduce to them in the subclass of downward maps homotopic to the identity. The relations in Theorem 2 incorporate the Lefschetz fixed point formula for the class of downward maps in a set of inequalities. Theorem 2 follows from Theorem 1, which contains more information in the coefficients other then the traces. Although the development can be given an abstract formulation in spectral homology theory, we shall confine discussion here to a concrete formulation sufficient for the application.

Suppose that $G$ is an abelian group finitely generated over the integers $J$ and that $f: G \rightarrow G$ is an endomorphism. Let $\phi_{f}(\lambda)$ denote the characteristic polynomial, a monic polynomial whose degree $n$ is the rank of $G$.

AMS 1970 subject classifications. Primary 57D70, 55C20; Secondary 58E05. 
Suppose that $G_{k}, k=0,1, \cdots$, is a sequence of finitely generated abelian groups and that $f_{k}: G_{k} \rightarrow G_{k}$ is a sequence of endomorphisms. The incomplete rational functions of $f=\left(f_{k}\right)$ are

$$
\Re_{f}^{q}(\lambda)=\prod_{i=0}^{q}\left[\phi_{f_{i}}(\lambda)\right]^{(-1)^{i}}
$$

If the sequence is finite, meaning $G_{k}=0$ for $k>N$, the characteristic rational function of $f$ is $Q_{f}(\lambda)=R_{f}^{N}(\lambda)$. This function is variously named in the references.

Suppose that $\left\{G_{k}, \beta_{k}\right\}$ with $\beta_{k}: G_{k} \rightarrow G_{k-1}$ and $G_{-1}=0$ is a homomorphism sequence of order two, such that homomorphisms $\beta$ and $f$ commute. With homology groups $H_{k}$ and induced homomorphisms $f_{* k}$ defined in the usual way, various authors have noted that, for finite sequences, $R_{f}(\lambda)=R_{f_{*}}(\lambda)$.

By the trace of a rational function written in the form $\lambda x-L \lambda x-1$ $+\cdots$, we shall mean the coefficient $L$.

Suppose that $X$ is a topological space and

$$
\varnothing=A_{0} \subset A_{1} \subset \cdots \subset A_{N}=X
$$

where each pair $\left(A_{i}, A_{i-1}\right)$ has finitely generated singular homology, trivial in dimensions exceeding $n$. Suppose that there is a map $f: X \rightarrow X$ with $f A_{i} \subset A_{i}$. Introduce the notations

$$
h_{k, i}=\left(f \mid\left(A_{i}, A_{i-1}\right)\right)_{* k} \quad \text { and } \quad h_{k}=\left\{h_{k, i} \mid i=0,1, \cdots, N\right\} \text {. }
$$

The latter acts on a direct sum and has the characteristic polynomial

$$
\psi_{k}(\lambda)=\prod_{i=1}^{N} \phi_{h_{k, i}}(\lambda)
$$

Reference [K-S] suggests a more sophisticated point of view, not explored here.

THEOREM 1. The polynomial $\phi_{f_{* k}}(\lambda)$ is a factor of the polynomial $\psi_{k}(\lambda)$. The incomplete rational function $\left(R_{f_{*}}^{q}\right)^{(-1)^{q}}$ divides $\left(Q_{h}^{q}\right)^{(-1)^{q}}$ with a polynomial quotient. Finally $R_{f_{*}}(\lambda)=R_{h}(\lambda)$.

The proof is computational. One observes that ${R_{f}}_{*}^{q}(\lambda)$ is global while $\mathcal{R}_{h}^{q}(\lambda)$ is assembled locally. In order to exhibit the local character, suppose further that $X$ is a compact differentiable manifold 
without boundary and $F$ is a smooth real valued function on $X$, all of whose critical points (for convenience in exposition and notation) are nondegenerate with no two at the same level. Suppose $f$ is a downward map, that is, a differentiable map such that $f x \neq x$ implies $F f x<F x$. Suppose

$$
a_{0}<c_{1}<a_{1}<\cdots<a_{N-1}<c_{N}<a_{N},
$$

where the $c_{i}$ are the critical levels of $F$, and set $A_{i}\left\{x \mid F x \leqq a_{i}\right\}$. Attach to the critical point $x_{i}$ of $F$ at level $c_{i}$ the index $k$ for which $H_{k}\left(A_{i}, A_{i-1}\right)=J$ and the order $d_{i}$, which is $\left(f \mid\left(A_{i}, A_{i-1}\right)\right)_{* k} 1$. The index and order of $x_{i}$ are determined on an arbitrary neighborhood of $x_{i}$.

Call the critical point $x_{i}$, with index $k$, of newly bounding type if the map $H_{k}\left(A_{i}, A_{i-1}\right) \rightarrow H_{k-1}\left(A_{i-1}\right)$ has kernel 0 . Call the map $f$ positive if the order of each critical point of newly bounding type is nonnegative.

We introduce the notations

$$
\begin{aligned}
A(q) & =\sum_{k=0}^{q}(-1)^{k} \operatorname{tr} f_{* k}, \\
D_{k} & =\sum_{i}\left\{d_{i} \mid x_{i} \text { has index } k ; i=1, \cdots, N\right\}, \\
B(q) & =\sum_{k=0}^{q}(-1)^{k} D_{k} .
\end{aligned}
$$

THEOREM 2. Corresponding to the downward map $f: X \rightarrow X$, the number $A(q)$ is the trace of the incomplete rational function $\mathbb{R}_{f_{*}}^{q}(\lambda)$ and $B(q)$ is the trace of $\mathcal{R}_{h}^{q}(\lambda)$. If $f$ is a positive map then

$$
(-1)^{q} B(q) \geqq(-1)^{q} A(q), \quad q=0,1, \cdots, n,
$$

with equality for $q=n$. The common value of $A(n)$ and $B(n)$ is the Lefschetz number of the map.

Added In Proof. The above account emphasizes the role of the map $f$. The function $F$ is used to distinguish the downward direction. One could achieve the same results with a different interpretation by emphasizing the critical point theory of $F$. Then $f$ enters in that the homology is computed with the polynomial ring $Q[f]$ over the rationals $Q$ as coefficient group.

\section{REFERENCES}

[F] E. Fadell, Recent results in the fixed point theory of continuous maps, Bull. Amer. Math. Soc. 76 (1970), 10-29. 
[K-S] J. L. Kelley and E. H. Spanier, Euler characteristics, Pacific J. Math. 26 (1968), 317-339. MR 41 \#5463.

[M] J. Milnor, Infinite cyclic coverings, Conference on the Topology of Manifolds (Michigan State Univ., East Lansing, Mich., 1967), Prindle, Weber \& Schmidt, Boston, Mass., 1968, pp. 115--133. MR 39 \#3497.

[S] S. Smale, Morse inequalities for a dynamical system, Bull Amer. Math. Soc. 66 (1960), 43-49. MR 22 \#8519.

Lehigh University, Bethlehem, Pennsylvania 18015 